\title{
La cadena de valor del sector confección
}

\author{
Sandrea, Maryana* \\ Boscán, Mariby* \\ * Economistas. Docentes-Investigadoras de la Universidad del Zulia. Instituto de \\ Investigaciones de la Facultad de Ciencias Económicas y Sociales. Departamento de \\ Estudios Macroeconómicos. Cursantes del Programa de Maestría en Gerencia de \\ Empresas. E-mail: marysandrea@yahoo.com y maribyboscán@yahoo.com
}

\section{Resumen}

El alto grado de competitividad empresarial sumado a los efectos del fenómeno de la globalización, abren nuevos retos y oportunidades para las empresas locales. Dentro de este contexto, el presente artículo tiene como objetivo identificar los componentes cualitativos de la cadena de valor para el producto líder del sector confección en el municipio Maracaibo del estado Zulia (Venezuela); por medio de un estudio de tipo descriptivo-transeccional, utilizando entrevistas semiestructuradas. De acuerdo a los resultados obtenidos las franelas constituyen el producto íder para la mayoría de las empresas entrevistadas. La construcción de la cadena de valor, mostró que la mayor parte de los gerentes del sector (72,73\% para 1999 y 66,67\% para el 2003) consideraban a la diferenciación del producto como el factor determinante en la decisión de compra del cliente. Al mismo tiempo, un 54,55\% en 1999 y un $81,82 \%$ en el 2003, identificó a la producción como la etapa clave en la generación de valor. Por lo que, se estiman como fundamentales las actividades primarias de operaciones y logística de entrada, así como las actividades de apoyo relativas al recurso humano, y al desarrollo de tecnologías. Finalmente, el estudio plantea como fuentes potenciales de ventajas competitivas, la optimización de actividades internas y el análisis de los nexos con proveedores, pudiendo aplicarse una estrategia competitiva de diferenciación con minimización del impacto en costos.

Palabras clave: Cadena de valor, competitividad, ventajas competitivas, actividades creadoras de valor, diferenciación. 


\title{
The Value Chain in the Clothing Sector
}

\begin{abstract}
The high level of competitiveness together with the effects of globalization have openned new challeneges and opportunities for local businesses. Within this context, this article focuses on identifying the qualitative components in the added value chaín for the leading products in the clothing sector in Maracaibo Municipality, Zulia State. This was done by means of a descriptive trans-sectional study using semistructured interviews. According to the results, T-shirts constitute the leading product for the majority of the firms interviewed. The construction of the value chain indicated that the majority of the managers in this sector $(72.73 \%$ in 1999 and $66.67 \%$ in 2003 ) consider differentiation of the product to be the determining factor in the purchase decision on the part of the consumer. At the same time, 54.55\% in 1999 and $81.82 \%$ in 2003 identified production as the critical stage in value generation. They indicated that the basic primary operational activities were entry logistics, and support activities related to human resources and technological development. Finally, the study proposed as potencial sources of competitive advantage, optimization of internal activities and the analysis of relations with suppliers, placing emphasis on a competitive strategy of differentiation with minimum cost impact.
\end{abstract}

Key words: Value chain, competitivity, competitive advantage, value creating activities, differentiation.

Recibido: 03-06-07. Aceptado: 04-03-02

\section{Introducción}

La vertiginosa naturaleza de los cambios, no distingue nacionalidades ni respeta fronteras, no existe sector industrial alguno, que pueda escapar a la globalización de los mercados, ni siquiera el tamaño y la distancia son armas eficaces para permanecer a salvo, se impone entonces la búsqueda de una gerencia proactiva y dinámica capaz de gerenciar atendiendo a la complejidad del mundo actual.

La investigación presentada gira en torno al enfoque del análisis de la cadena de valor como herramienta susceptible de ser utilizada para mejorar el desempeño competitivo de una organización, pues el referido enfoque, posee un gran potencial estratégico para encontrar fuentes de ventajas competitivas optimizando la creación y/o desarrollo de las mismas. En Venezuela, los sectores del calzado y la confección constituyen áreas 
prioritarias para la política de reactivación industrial, en vista del gran número de pequeñas y medianas empresas que se dedican a esta actividad y al volumen de puestos de trabajo que generan, sobre todo en el caso del empleo femenino, de allí la intención de aplicar el referido enfoque al sector confección.

Como objetivo general esta investigación plantea: Identificar los componentes cualitativos de la cadena de valor para el producto líder del sector confección en el municipio Maracaibo del estado Zulia. Para alcanzar dicho objetivo se identifican los elementos internos y externos de la cadena, las actividades creadoras de valor dentro y fuera de las empresas del sector (en términos generales), así como las fuentes potenciales de ventajas competitivas del producto líder del sector a nivel local, para finalmente, determinar la estrategia competitiva genérica, conveniente para desarrollar las ventajas competitivas locales.

Se consideran como variables principales del estudio los componentes de la cadena de valor genérica, así como las fuentes potenciales de ventajas competitivas, las cuales vienen definidas por las áreas y actividades (dentro y fuera) de la empresa, donde es posible encontrar ventajas competitivas. Para la ejecución de la investigación, se estableció como periodo de análisis los meses comprendidos entre Julio y Septiembre del año 1999 y del 2003, refiriéndose la misma sólo al municipio Maracaibo del estado Zulia en Venezuela.

El tipo de estudio planteado es de carácter descriptivo-transeccional, ya que propone presentar los elementos cualitativos de la cadena de valor para momentos específicos en el tiempo, siendo realizado bajo una visión gerencial y económica. La población objeto de estudio está representada por empresas que confeccionan todo tipo de ropa (casual, de vestir, franelas, camisas, faldas, pantalones, etc.), es decir, empresas del sector confección ubicadas geográficamente (al menos su área de producción) en el referido municipio, en tanto que la unidad de análisis esta constituida por la gerencia de dichas empresas.

Según las estadísticas proporcionadas por la cámara de industriales del estado Zulia, los registros de información telefónica así como por una revisión realizada por los investigadores del Instituto de Investigaciones FCES-LUZ, existían para 1999 en el municipio Maracaibo un total de 68 empresas pertenecientes al sector confección. Sin embargo, para ese entonces sólo fue posible entrevistar a los gerentes de once empresas. Para el año 2003 se encontró que el sector está constituido por un total de 62 empresas según la base de datos del programa "Factores de competitividad de las pequeñas y 
medianas empresas de la región zuliana 2003" (Sandrea y Boscán, 2003). A fin de realizar una comparación con los resultados obtenidos en 1999, se consideró conveniente efectuar un seguimiento a las once empresas estudiadas anteriormente, para así constatar si prevalecen las tendencias reveladas en aquella oportunidad, dado que las mismas fueron entonces barómetros eficientes para el resto del sector. De las once empresas entrevistadas en 1999 sólo fue posible localizar seis, ya que las cinco restantes desaparecieron como consecuencia de la difícil situación político-económica que atravesó Venezuela a finales del año 2002.

Puesto que el marco poblacional definido por esta vía no es exhaustivo, se considera que el estudio realizado es cualitativo (tendencial), aunque se utilicen técnicas cuantitativas como la entrevista semiestructurada, razón por la cual los resultados del estudio no serán concluyentes, pero si permitirán revelar tendencias interesantes sobre el comportamiento de las variables estudiadas.

\section{Consideraciones teóricas sobre el enfoque de la cadena de valor}

La cadena de valor estudia la creación de valor dentro y fuera de la empresa, hasta llegar al consumidor final y viceversa. Consiste en "el conjunto interrelacionado de actividades creadoras de valor (diseñar, fabricar, vender y distribuir.) que se extienden durante todos los procesos, desde la consecución de fuentes de materias primas para proveedores de componentes hasta que el producto terminado se entrega finalmente en las manos del consumidor" (Shank y Govindarajan, 1997: 16). Este enfoque coloca en primer plano "el valor para el cliente" que la empresa puede brindarle en el presente y futuro (Porter, 2000).

Según esta definición, cada empresa tiene una cadena de valor exclusiva para cada producto, sin embargo, este enfoque posee un potencial consolidante, es decir al tener la cadena para la mayor parte de las empresas que conforman un sector, se puede consolidar la cadena de valor para el sector completo. De este modo todas las empresas que conforman una industria tendrán una cadena de valor que resalte aquellas actividades comunes (tipos de materias primas, aspectos básicos de los productos, servicio al cliente, entre otras).

La cadena de valor puede representarse en forma cualitativa, identificando las etapas de elaboración que agregan valor al producto, dentro y fuera de la empresa hasta llegar al consumidor final, o en forma cuantitativa, cuando se especifican adicionalmente los costos, ingresos y activos asociados a cada actividad de valor. Para construir la cadena de 
valor cualitativa se considerará la metodología de Shank y Govindarajan (1997) expuesta en su obra Gerencia Estratégica de Costos, la cual plantea la identificación de las actividades que crean valor dentro de la cadena, permitiendo establecer las fuentes de ventajas competitivas.

De acuerdo a esta metodología, las actividades de valor comprenden las acciones medulares que realiza una empresa para crear un producto o servicio valioso para sus compradores. El título: "Actividad de valor" es una designación arbitraria que depende fundamentalmente de la relación de las actividades de la empresa con la generación de valor para el comprador. Así mismo, para efectos de la construcción de la cadena, las actividades creadoras de valor se clasifican en primarias o de apoyo (Porter, 1998: 56$57)$.

Una cadena de valor genérica comprende tres elementos básicos: Las actividades primarias, de soporte y el margen. Las actividades primarias, están relacionadas con la creación física del bien o servicio (consecución de materias primas, producción, comercialización, mercadeo, así como los servicios de post-venta), incluyen logística entrante, operaciones, logística saliente, comercialización/ventas y servicios; mientras que, las actividades de apoyo se refieren a aquellas tareas funcionales que permiten ejecutar las actividades primarias (prestan respaldo general y especializado), pudiendo contarse entre otras: abastecimiento, desarrollo de tecnologías, gestión de recursos humanos, e infraestructura de la empresa (finanzas, contabilidad, gerencia y asesoría legal). Finalmente, se denomina margen a la diferencia entre el valor total y los costos totales en que incurre la empresa para desempeñar las actividades creadoras de valor (Ayala y Arias, 2003).

La cadena de valor no responde únicamente a aspectos internos de la empresa, no es estática ni unidireccional, tampoco constituye una solución única, pueden crearse tantas como sean necesarias, las cuales deben ser altamente integradas, activándose principalmente del lado de los clientes. Desarrollar la cadena de valor es un proceso progresivo y complejo que empieza en el cliente, ya que es el usuario final quien define el valor, y se mueve hacia la empresa, sus proveedores y finalmente proveedores de proveedores. Este proceso implica intercambios de información y vínculos en el flujo de productos entre clientes, proveedores y terceros (La londe, 2000).

A partir de la identificación de la cadena de valor, una empresa puede localizar ventajas competitivas sostenibles mediante dos formas: controlando las causales de costo mejor que los competidores y reconfigurando la cadena. Por lo que, “...El análisis de la cadena 
de valor es esencial para determinar dónde, exactamente, en la cadena del cliente se puede aumentar el valor, o rebajar los costos..." (Shank y Govindarajan, 1997: 65).

Las fuentes de ventajas competitivas bajo la óptica de la cadena de valor pueden provenir de: los eslabones entre las actividades de valor, la ejecución de las mismas, las diferencias entre la cadena de valor de la empresa y las de los competidores, y el análisis de la cadena de la empresa en el contexto del sistema de valor total del producto. Este concepto, considera que la empresa es parte de un complejo de actividades ejecutadas por diversos actores (proveedores, distribuidores y clientes), por tanto es posible analizar la cadena de valor de los proveedores, de los canales y de los compradores (Ayala y Arias, 2003).

La construcción y análisis de la cadena de valor permite descubrir y desarrollar ventajas competitivas en términos de las cuales se construyen estrategias empresariales. Sin embargo, quizás la decisión estratégica más importante que debe tomar una empresa consiste en decidir que tipo de ventaja competitiva quiere obtener. Según Porter (2000) el encaje de las actividades en la cadena constituye en ś mismo la fuente principal de ventajas competitivas, resaltando la importancia del sistema de actividades. Así mismo, las empresas pueden desarrollar ventajas competitivas basándose en estrategias como: liderazgo general en costos, diferenciación, y enfoque, nicho o alta segmentación (objetivo limitado), dependiendo la elección de una u otra, de la estructura del sector industrial al que pertenece una empresa determinada.

El liderazgo en costos es una estrategia diseñada para segmentos de mercado sensibles a los precios, en los cuales se ofrece un producto similar a un precio bajo. En este tipo de estrategia una empresa se propone ser el productor de menores costos dentro del sector industrial al que pertenece. La estrategia de diferenciación consiste en brindar al comprador un valor superior, en términos de calidad, características especiales y servicios post-venta, bajo el supuesto de que el mercado recompensará su exclusividad pagando un precio mayor. Por último la estrategia de enfoque consiste en concentrarse en un grupo de compradores en particular, en un segmento de la línea del producto o en un mercado geográfico, ofreciendo una atención intensiva para los clientes de modo de asegurar su preferencia.

La evaluación de los resultados de la cadena de valor puede realizarse atendiendo a diversos criterios: costo (usualmente se utiliza el costo basado en actividades "ABC"), inventario (ya que éste representa un valor considerable en términos de activos corrientes), velocidad (tiempo transcurrido entre la generación de una orden y la entrega 
del pedido al cliente), y finalmente considerando el criterio financiero (condiciones y tiempo de pago a los proveedores) (La londe, 2000).

\section{La industria de la confección venezolana}

Se entiende como sector confección, aquel que se ocupa de la transformación (diseño, corte y costura) de la tela, cuero, pieles y otros materiales provistos por la industria textil, en una prenda lista para ser usada por el consumidor final como indumentaria de vestir (excepto calzado), asimismo, incluye la fabricación de sombreros, adornos y accesorios. Este sector esta definido como grupo 322 dentro de la clasificación industrial internacional uniforme (CIIU) de las Naciones Unidas (Romero et al., 2000).

Dependiendo de su orientación estratégica, la industria de la confección puede ser intensiva en mano de obra, en cuyo caso su expansión se orienta a la existencia de recurso humano abundante y a bajo costo; recibiendo protección de los países cuyos gobiernos la utilizan como instrumento empleador (Romero et al, 2000). Tambi én puede ser una industria altamente diferenciada (producción exclusiva), en la cual se manejan bajos volúmenes de producción colocados a precios altos. Como ocurre en Italia y Alemania, dónde la industria de la confección carece de un alto potencial empleador.

En Venezuela, a pesar de que el sector confección posee una larga historia, éste no ha logrado un desarrollo a gran escala, evidenciando en todo su proceso histórico la falta de cohesión, visión y objetivos comunes entre las empresas. En contraste con las referidas características desfavorables, que han sido una constante en la evolución del sector, destacan iniciativas recientes por parte de los empresarios y representantes del gobierno para establecer una visión del negocio compartida, identificar las bases para la competencia internacional (objetivos estratégicos), y establecer acuerdos formales para el intercambio de información, capacitación del personal, financiamiento, entre otras áreas (Monitor Company, 1997).

Actualmente el sector confección venezolano atraviesa por múltiples problemas, tanto a nivel estratégico como operacional; por una parte se hace evidente la carencia de una visión nacional del negocio así como del diseño y ejecución de estrategias eficientes para alcanzar una posición competitiva internacional, mientras que por otro lado, a nivel operativo la industria en los últimos años ha confrontado graves dificultades para aumentar sus niveles de ventas y producción así como mejorar otros aspectos importantes para el sector. 
Esta industria se caracteriza por presentar un alto grado de fragmentación de la oferta e informalidad en su estructura empresarial (Romero et al., 2000), destacando la presencia de una gran multitud de pequeñas microempresas familiares y una importante concentración geográfica de las empresas alrededor de la región capital, además presenta un bajo nivel de demanda interna; hipersensibilidad del consumidor ante el precio de los productos confeccionados; uso intensivo de mano de obra; alto grado de rivalidad interna entre las empresas del sector; bajo nivel de productividad laboral (en comparación con los estándares internacionales); altos niveles de costos con referencia a la materia prima así como una evolución desfavorable de la balanza comercial sectorial. Entre los principales productos del sector se encuentran: ropa interior masculina y femenina, tejido de punto femenino y masculino, camisas, jeans, corbatas, uniformes, ropa de bebes y alta moda.

En cuanto a la balanza comercial, el sector confección venezolano presenta casi constantemente un saldo negativo, fundamentado en el bajo nivel de exportaciones. A nivel internacional los competidores más importantes para los productos venezolanos son China, Corea, Italia, México, Colombia y Brasil. Asimismo, el parque industrial venezolano evidencia un bajo nivel tecnológico, caracterizado por la escasa automatización de las fases del proceso productivo y un rezago de aproximadamente quince años, en relación a los niveles tecnológicos internacionales. Al tiempo que, muestra una carencia de personal calificado, así como de centros adecuados para la capacitación (INE, 1997).

En los últimos meses el gobierno venezolano ha tomado medidas en materia económica que directa o indirectamente han influido en el sector confección, entre las cuales destacan: a) el establecimiento del control de cambio, por una parte ha impedido la importación de productos confeccionados para el consumo final, lo cual facilita la colocación de la producción nacional, pero al mismo tiempo ha dificultado la obtención de materias primas importadas, por lo que el efecto neto es ambiguo; b) Las normas de etiquetado, que consisten en mencionar en las etiquetas de las prendas de vestir las características de las telas utilizadas en su elaboración, brindan al consumidor una variable objetiva para evaluar la calidad del producto; c) El aumento en las compras gubernamentales, y d) el financiamiento especial. Se espera que estas medidas tengan un efecto positivo en el sector en cuanto al uso de su capacidad instalada (Fernández, 2003).

\section{La industria de la confección zuliana}

Aunque la región zuliana está fuera de la zona de alta concentración de empresas de la confección, ofrece importantes ventajas comparativas para el fortalecimiento de este tipo de industria, derivadas de ser una zona fronteriza en contacto con una industria textilera 
de mayor desarrollo y tradición que la venezolana, como lo es el mercado textil colombiano. A nivel regional, el sector confección exhibe fundamentalmente las mismas características que presenta a nivel nacional, con la diferencia que el número de empresas es significativamente inferior al de la región capital, por tanto los volúmenes de producción, ingreso y nivel de empleo, son también limitados.

\subsection{I dentificación del valor para el mercado meta}

Las fuentes potenciales de ventajas competitivas están íntimamente relacionadas con los aspectos del producto líder que generan valor para el mercado meta y las actividades internas y externas relacionadas con dichos aspectos, pues ello determina las etapas clave para la creación de valor así como el tipo de estrategia competitiva a desarrollar.

En este sentido, para el año 1999 se encontró que la mayor parte de las empresas dedicadas a la fabricación de indumentaria de vestir (excepto calzado) presentaban como producto líder, con mayor frecuencia relativa, las franelas $(36,37 \%)$, manteniéndose el mismo para el año 2003 , con un $66,67 \%$ de los casos estudiados.

En cuanto a los aspectos del producto líder que generan valor para el mercado meta, para el año 1999, un poco más de la mitad de los gerentes entrevistados $(54,55 \%)$, fue capaz de realizar una descripción concreta (elementos puntuales) de estos aspectos, mientras que un $27,27 \%$ de los entrevistados realizó una descripción vaga, referida sólo a aspectos generales, por lo que podría decirse que el $81,82 \%$ conocen, a nivel puntual o general, los aspectos generadores de valor de su producto líder, logrando identificar las etapas claves para la creación de valor en la cadena. Sólo un $18,18 \%$ del total entrevistado no respondió este indicador. Esta tendencia se mantiene para el año 2003, donde a pesar de que sólo sobrevivieron seis de las once empresas estudiadas anteriormente, el 66,67\% realizó una descripción concreta de los aspectos puntuales que generan valor para el mercado meta, mientras que el 33,33\% efectuó una descripción basada sólo en aspectos generales, con lo cual se encontró que el $100 \%$ del total de los gerentes entrevistados tiene conocimiento de los aspectos del producto líder que generan valor para el mercado.

Adicionalmente, de acuerdo con los resultados del estudio realizado pudo constatarse que, la mayor parte de los entrevistados, un $72,73 \%$ para 1999 y un 66,67\% para el 2003 considera que su mercado meta, valora fundamentalmente algún atributo diferenciador en su producto (marca, calidad y diseños, entre otros), a la hora de tomar la decisión de compra, es decir, que a pesar de la hipersensibilidad del consumidor venezolano hacia el 
precio, los confeccionistas maracaiberos opinan que sus clientes valoran más la diferenciación del producto, ello puede deberse a que los niveles de costos son similares entre los empresarios (rigidez en costo de la materia prima), que sumado a la depresión de la demanda interna, obliga a ofrecer un producto a precios parecidos a los de los competidores nacionales y extranjeros, valiéndose de la diferenciación para inclinar favorablemente la decisión de compra del consumidor (Ver Gráfico 1).

\section{Gráfico 1}

Identificación del valor para el mercado meta del sector confección en Maracaibo

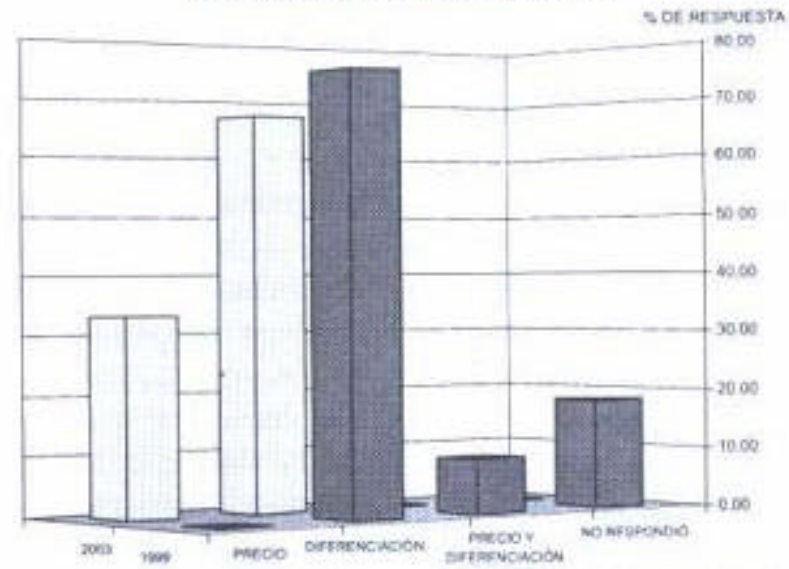

Fuente: Elaboración Propia Considerando los datos obtenidos a partir de las entrevistas realizadas a empresarios del sector (1999 y 2003)

\subsection{Actividades y etapas clave en la creación de valor para el mercado meta}

En relación a la generación de valor en actividades internas, para 1999 un 81,82\% del total entrevistado fue capaz de identificar (en forma puntual o general) las actividades internas generadoras de valor, mientras que para el 2003 el resultado fue de un 100\%. Además, un 54,55\% para 1999 y un 83,33\% para el 2003, consideran a las actividades relacionadas con el proceso de producción, como las más relevantes para la generación de valor. Entre estas destacan: el diseño, corte y costura (combinación de colores, acabado final de la prenda), y la investigación de las tendencias de la moda (compra de revistas actualizadas). Este resultado corresponde a las características propias de empresas que fabrican bienes.

En contraste con el resultado obtenido en la identificación de actividades internas relacionadas con lo que el mercado meta valora, el análisis de las actividades externas para 1999 mostró un alto nivel de desinterés por parte de los entrevistados, tanto es así que, la mayor parte de los mismos $(54,55 \%)$ no respondió la pregunta vinculada a este tópico, al tiempo que un 27,27\% del total entrevistado consideró que "todo era interno", desestimando de esta forma, la participación de las actividades externas en la generación 
de valor para sus clientes, lo que equivale a decir que, por ejemplo, la calidad de la materia prima aportada por los proveedores, la oportunidad de entrega, la actuación de los canales de distribución no afecta el "valor" del producto para el cliente. Mientras que para el 2003 se evidenció un cambio de actitud en cuanto al impacto de las actividades externas sobre la generación de valor para el mercado meta; el 83,33\% de los entrevistados respondió a este indicador, considerando que algunas características de las telas aportadas por los proveedores (diseños, calidad, precio, diferenciación, entre otras), eran importantes para la generación de valor del producto final.

De acuerdo a los resultados obtenidos tanto para 1999 como para el 2003, destacan como etapas claves en la generación de valor: Producción, supervisión y control de calidad, aunque hablando en términos generales, las dos últimas pueden englobarse dentro de las actividades del proceso productivo, por lo que es posible concluir que según la información recabada, la producción dentro del sector confección del municipio Maracaibo del estado Zulia, se considera como la etapa principal para la generación de valor.

\subsection{Las fuentes de ventajas competitivas}

Según los resultados del estudio realizado, la gerencia del sector confección del municipio Maracaibo del estado Zulia, identifica como fuentes potenciales de ventajas competitivas: Mantenimiento de precio bajo (relación precio-calidad) 27,27\% para 1999 y $33,33 \%$ para el 2003; diseño, acabado y confección de la prenda 22,72\% en 1999 y $50 \%$ en el 2003, servicios post-venta (9,09\% sólo fue considerado en 1999) y calidad de las telas (16,67\% sólo fue considerado en el 2003). Cabe destacar, que para 1999 el 36,37\% de los entrevistados no respondió a esta variable, mientras que en el 2003 todos los gerentes entrevistados identificaron las fuentes de ventajas competitivas para su producto líder, lo cual es una evidencia de la toma de conciencia de los empresarios sobre la importancia del estudio de la competitividad del producto líder.

Una vez identificados los aspectos que el mercado meta valora, las actividades internas y externas relacionadas con éstos, habiendo además establecido las etapas claves para la generación de valor, así como la opinión de la gerencia sobre sus fuentes de ventajas competitivas, se tiene que, en este caso, la estrategia competitiva más factible es la de diferenciación, pero sí ésta última resulta demasiado costosa, traerá consigo la muerte súbita de la empresa, dada la sensibilidad del consumidor maracaibero hacia el precio de los productos, es decir, se impone la creatividad, y el ingenio para encontrar elementos diferenciadores que no impliquen aumentos significativos de costos, donde la ganancia 
adicional esté representada en un incremento de la demanda (impulsado por el atractivo diferenciador).

Por lo tanto, las ventajas competitivas para el sector confección en Maracaibo pueden provenir fundamentalmente de: a) los nexos entre actividades operativas que implican optimización de las actividades internas (mejora en tiempos de procesos) manteniendo una estructura de costos bajos (proceso productivo) que permita lograr diseños Novedosos, excelente acabado en cuanto a la confección de la prenda, óptimos servicios post-venta y una excelente atención al cliente; b) Nexos con los proveedores, que optimicen la calidad de la materia prima, y entrega justo a tiempo de ésta (coordinación), manteniendo costos similares a los de los competidores; y c) Nexos con los canales de distribución que permitan una adecuada accesibilidad al producto y servicios post-venta; utilizando controles de calidad en todas las fases del proceso productivo (ver Diagrama 1 ).

\subsection{El proceso productivo}

Las empresas del sector confección del municipio Maracaibo del estado Zulia poseen una estructura organizacional bastante sencilla, frecuentemente distribuida en las áreas clásicas: Administración, producción y ventas; perteneciendo la mayor parte de ellas a la pequeña y mediana industria (clasificación de acuerdo al criterio del Instituto Nacional de Estadísticas de 5 hasta 100 personas empleadas).

El proceso de producción típico en la industria de la confección comprende dos fases: a) Preensamblado y b) Ensamblado. El preensamblado implica la creación de un diseño según los gustos y requerimientos del mercado meta, que luego es traducido a un prototipo, en el caso de las marcas "compradas" esta etapa se suprime; posteriormente, cada parte del modelo es reproducida en todas las tallas, patrones o moldes de cartón, luego se desenrolla la tela, se coloca sobre la mesa de corte, se trazan los patrones y se corta ésta. El ensamblado, incluye las actividades de etiquetado, organización de piezas previamente cortadas, costura, acabado y planchado de prendas.

Tradicionalmente, el proceso productivo del sector confección requiere de un alto componente de mano de obra que puede variar de acuerdo al grado de desarrollo tecnológico de la industria.

En la actualidad los adelantos tecnológicos introducidos al sector, como: diseño asistido por computadora, patrones computarizados, cortadoras de control numérico, cortadoras a base de rayos láser o chorro de agua, carros automáticos para desenrollar la tela, etc., 
han permitido alcanzar una mayor eficiencia en el proceso productivo, eliminando incluso algunas de sus fases manuales, lo cual contribuye a la reducción del desperdicio en la producción. A pesar que a nivel internacional el uso de éstos avances está bien extendido, los mismos han tenido una penetración escasa en Venezuela, debido entre otras causas al limitado nivel de inversiones destinadas a la adquisición y desarrollo de nuevas tecnologías.

En términos generales, el proceso productivo de las empresas del sector confección del municipio Maracaibo del estado Zulia, utiliza en forma intensiva el recurso humano, realizando manualmente la mayoría de las fases, destaca la escasa utilización de maquinaria de alta tecnología y la presencia de etapas tradicionales en todo el proceso, tales como el diseño, extendido de la tela y trazado manual (Diagrama 1).
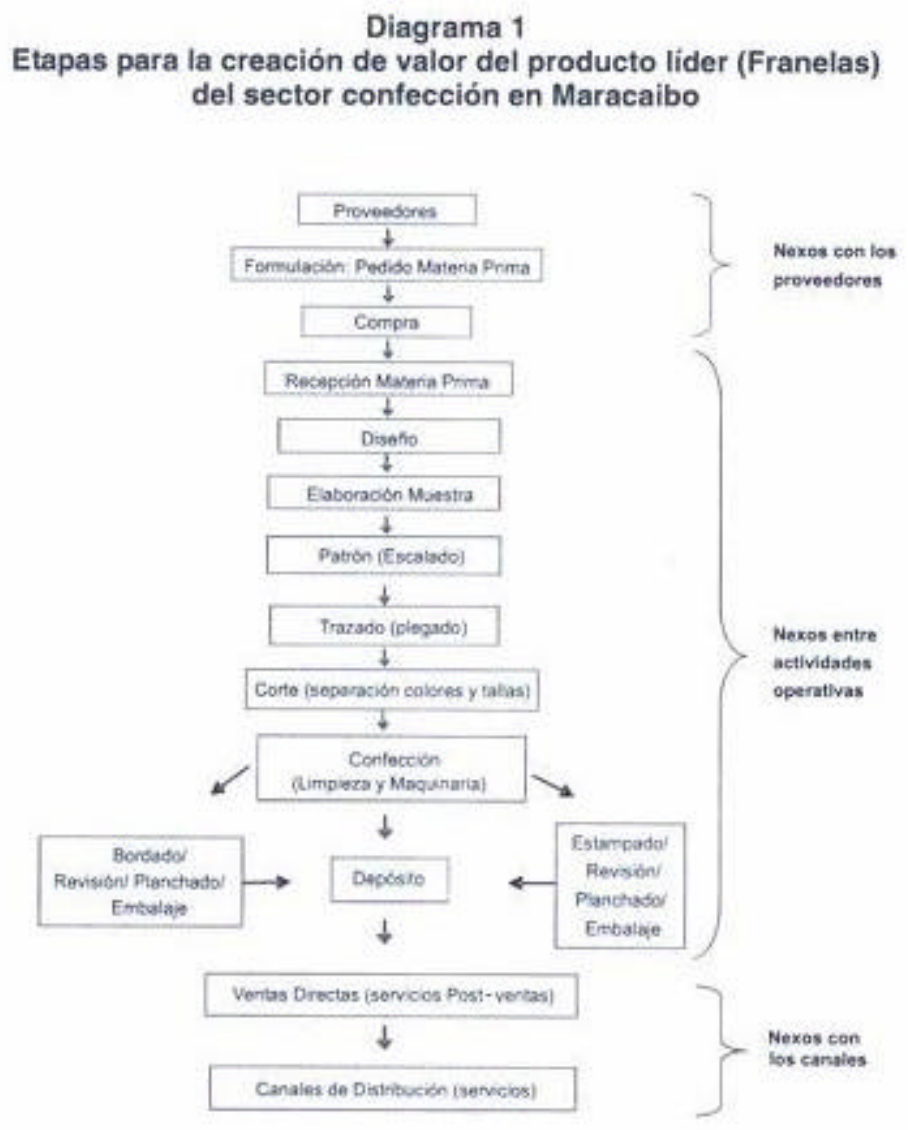

Fuento: Elaboracion Propia. Dalos obtenides a partir de las entrevistas realizadas a ompresarios del sector (1999 y 2000).

\subsection{La cadena de valor genérica de la industria}

La construcción de la cadena de valor, permite visualizar las actividades primarias y de apoyo, vitales para cualquier empresa. Según Porter (1998) el abastecimiento, el desarrollo de tecnología y la administración de recursos humanos pueden asociarse con 
actividades primarias específicas, sin embargo, la infraestructura de la empresa no está asociada con actividades primarias particulares, sino que apoya a la cadena entera. En el caso de la industria de la confección, específicamente empresas fabricantes de franelas, las operaciones y la logística de entrada, figuran como las actividades primarias más relevantes, al tiempo que el recurso humano y tecnológico asociado a las mismas, fueron identificadas como las actividades de apoyo más importantes (Ver Cuadro 1).

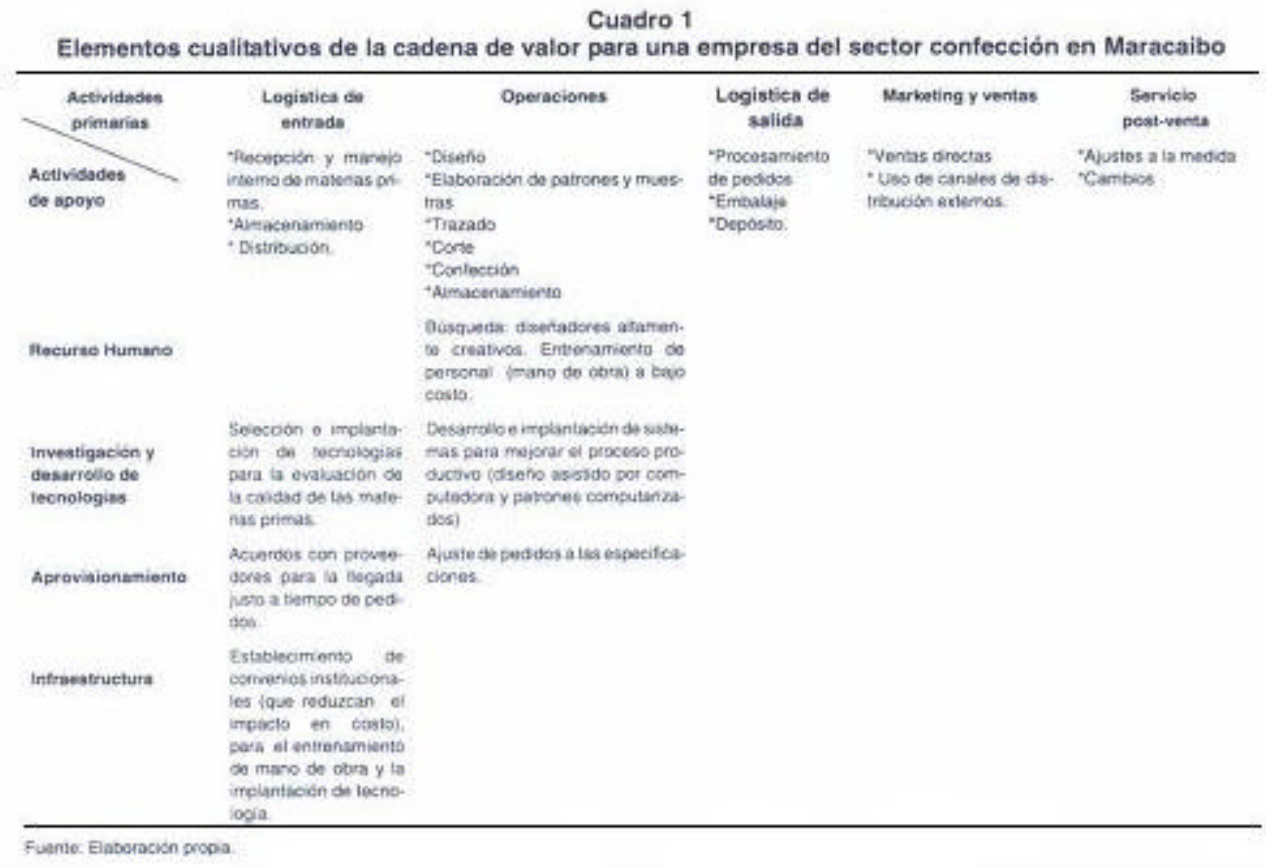

\subsection{Estrategias competitivas del sector confección en Maracaibo}

A pesar de ser Venezuela un país con relativo éxito en el diseño de prendas de vestir, especialmente trajes de baño para damas (Monitor Company, 1997), para 1999 los gerentes del sector confección del municipio Maracaibo del estado Zulia, no identificaron la diversificación como elemento preponderante para el diseño de la estrategia competitiva del producto líder; el $18,18 \%$ de los gerentes entrevistados manifestaron que el costo era el elemento clave para el diseño de estrategias competitivas. Un $27,27 \%$ del total entrevistado, analiza más bien una combinación de precio y diversificación, al tiempo que, un $36,37 \%$ de los gerentes no respondieron a este indicador. En tanto que en el 2003 un $50 \%$ de los entrevistados consideró a la diversificación como el elemento clave para el diseño de estrategias competitivas, un 25\% manifestó que analizaban una combinación precio-diversificación, al tiempo que el restante $25 \%$ no respondió a este indicador (Gráfico 2). 
Gráfico 2

Elemento clave para el diseño de la estrategia competitiva del sector confección en Maracaibo

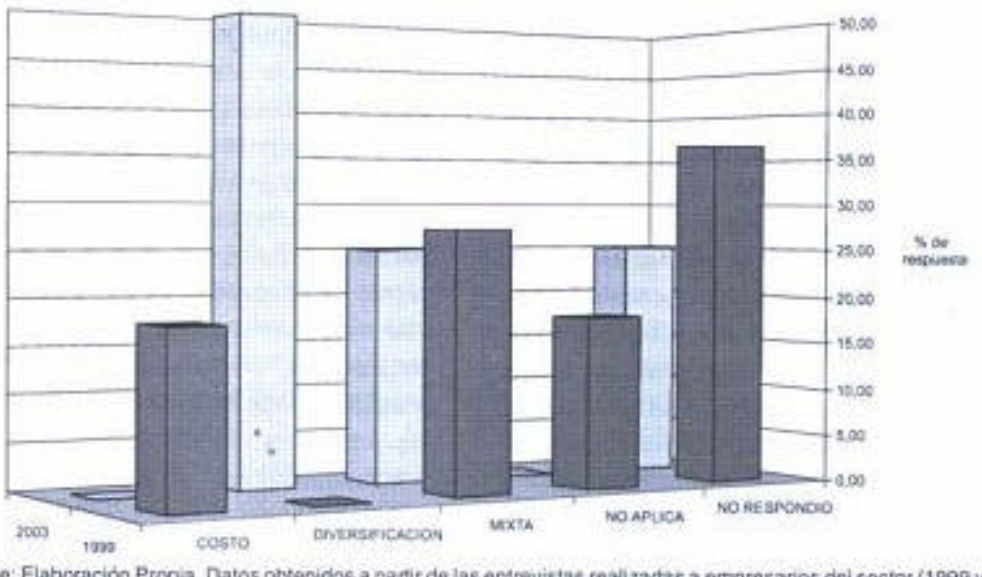
2003)

Estos resultados permiten afirmar que recientemente los empresarios del sector han concientizado la importancia de la diversificación en el diseño de estrategias competitivas, con lo que se pone de manifiesto el interés por el estudio de las mismas. En este sentido, cabe destacar que para alcanzar un nivel de competitividad internacional, las empresas venezolanas han de plantearse desarrollar estrategias competitivas diversificadas, pues los costos internos aun son más elevados que en los países asiáticos, estas estrategias deben estar dirigidas especialmente hacia los mercados europeos, a nivel regional la estrategia competitiva de diversificación a bajo costo parece ser la opción más viable.

Por otro lado, una estrategia de liderazgo en costo es recomendable para la producción masiva de bienes dirigidos hacia segmentos grandes y de mayor homogeneidad de demanda. Allí el precio, garantizado por la escala de producción, es un elemento de competencia, que junto a la calidad y oportunidad de entrega, privan sobre el diseño y el apego a la moda. Estas estrategias son viables especialmente para países con abundancia de mano de obra, la cual prevé un costo salarial bajo como sustento de la competencia vía precios. Esta parece haber sido en el pasado la estrategia seguida por algunos países asiáticos. Sin embargo, en el caso venezolano a pesar de la disminución del salario real, la carencia relativa de mano de obra calificada, junto con la atomización de la oferta así como los reducidos volúmenes de producción por empresa, dificultan el desarrollo de este tipo de estrategia basada en la producción a gran escala.

Según los estudios realizados por la compañía Monitor Company (1997) el problema fundamental del sector confección venezolano, ha sido una falta de visión tanto por parte del sector privado como del público respecto a ¿dónde quiere llegar en el futuro, y en qué 
se quiere convertir? La industria de la confección venezolana en su conjunto tiene un gran potencial para adoptar una estrategia diferenciada, dirigida por el sector privado con apoyo gubernamental similar en algunos aspectos al modelo italiano. Asimismo, los referidos estudios, sobre la competitividad del sector confección, evidencian que Venezuela elabora productos a precios superiores en comparación con países como México y China. En cuanto a la estructura de costos del sector, el renglón referido a la materia prima (tela) representa el mayor peso, la cual se encarece debido a la característica oligopólica del mercado textil, mientras que el costo de la mano de obra no es limitativo para la competitividad del sector. A pesar de la caída en el costo laboral, el impacto de la tela en la estructura de costo unido a las deficiencias en plataforma operativa dificulta la posibilidad de desarrollar estrategias competitivas de liderazgo en costo, como las creadas en Corea.

Por otro lado, sí bien los bajos salarios son una oportunidad para generar ventajas competitivas en costos, éstos no deben permanecer estancados, pues a largo plazo se convertirán en frenos para el despegue competitivo. Un recurso humano desmotivado por la permanencia de bajos salarios, no se esfuerza por ser más productivo, y es campo fértil para generar problemas laborales en la empresa.

Finalmente, una estrategia de diferenciación de espectro limitado, basada en la producción flexible de pequeños lotes dirigidos a segmentos diferenciados de demanda, donde el diseño y el apego a la moda junto a la calidad y oportunidad de entrega, son los elementos fundamentales de la competencia, pareciera viable en el caso venezolano.

\section{Conclusiones}

Entre las principales características del sector confección venezolano destacan: alta concentración geográfica de las empresas (región capital); elevado grado de fragmentación de la oferta (preponderancia de micro y pequeñas empresas); bajo nivel de demanda interna; hipersensibilidad del consumidor ante el precio de los productos confeccionados; alto nivel de informalidad en la estructura empresarial; uso intensivo de mano de obra; alto grado de rivalidad interna entre las empresas del sector; bajo nivel de productividad laboral (en comparación con los estándares internacionales); altos niveles de costos con referencia a la materia prima así como una evolución desfavorable de la balanza comercial sectorial. Esta combinación de características permite visualizar un sector cargado de problemas y al mismo tiempo lleno de oportunidades, ya que la reactivación del mismo, podría constituirse en la piedra angular para el desarrollo de una política de impulso al empleo industrial. 
Aunque el municipio Maracaibo del estado Zulia, se encuentra fuera del área de alta densidad empresarial con respecto a este sector, ofrece como localidad geográfica importantes ventajas comparativas para el desarrollo de este tipo de industria entre las que destacan facilidad de acceso al mercado de la confección colombiano, del cual pudiera proveerse de materia primas de excelente calidad, así como aprovechar, vía proceso de benchmarking, la experiencia confeccionista de las empresas de ese país y la existencia de un puerto comercial para el intercambio marítimo.

Luego en cuanto a las variables seleccionadas los resultados del estudio realizado permitieron identificar las siguientes tendencias:

Las franelas resultaron ser el producto líder para la mayor parte de las empresas estudiadas tanto en 1999 como en el 2003, al tiempo que casi la totalidad de los gerentes entrevistados fueron capaces de identificar a nivel puntual o general los aspectos clave del producto líder que generan valor para el mercado meta, considerando a la diferenciación del producto como el elemento central.

Las actividades internas que se identificaron como las más relevantes para la generación de valor comprenden, el diseño (que incluye la selección y la combinación de colores), el corte, la costura (acabado final), agrupando dentro de éstas dos últimas actividades el control de calidad en el trazado y la confección, la investigación sobre tendencias de la moda, el entrenamiento y supervisión del personal especialmente en cuanto al uso del equipo productivo, el control de calidad en la adquisición de materias primas, y finalmente la atención al cliente y los servicios post-venta. Por tanto, el proceso productivo destaca como la etapa clave en la generación de valor.

De acuerdo a las ideas expresadas en el párrafo anterior, las oportunidades de generación de valor para el producto líder con mayor frecuencia relativa en el municipio Maracaibo del estado Zulia, se centran en: a) selección de materia prima de alta calidad (incorporar sistemas para caracterizar la calidad de la materia prima), b) entrega justo a tiempo de la materia prima, c) mejora en los tiempos de procesos y costos, d) elaboración de diseños novedosos, e) brindar excelente acabado y atención al cliente, f) mejorar los sistemas de distribución y accesibilidad al producto, entre otras. En cuanto a las actividades externas, se incluyen aquellas relacionadas con los proveedores: Coordinación (entrega justo a tiempo de materia prima), calidad (materia prima uniforme, ajustada a las especificaciones técnicas solicitadas), y disponibilidad de materiales informativos sobre tendencias de la moda. Con referencia al canal se incluye la accesibilidad al producto y los 
servicios post-venta. Mientras que las relativas al cliente, comprenden la actitud de éste hacia el producto (determinantes de la decisión de compra).

La gerencia del sector confección del municipio Maracaibo del estado Zulia, identifica como las principales fuentes de ventajas competitivas: la optimización de las actividades internas (diseño, corte y costura de prendas a precios bajos, servicios post-venta), así como el estudio de los nexos con los proveedores (calidad de las telas y oportunidad de entrega). Es importante resaltar, que para el 2003 el 100\% de los gerentes entrevistados identificaron las fuentes de ventajas competitivas para su producto líder, mientras que en 1999 un $36,37 \%$ no respondió a este indicador, lo cual puede considerarse como una señal positiva de toma de conciencia por parte de los gerentes del sector sobre la competitividad de su producto líder.

Las empresas del sector confección del municipio Maracaibo del estado Zulia, realizan manualmente la mayor parte de las etapas de su proceso productivo, por lo que utilizan intensivamente el recurso humano al tiempo que las maquinarias utilizadas revelan un importante grado de obsolescencia del parque industrial. En referencia a la cadena de valor de las empresas fabricantes de franelas del municipio Maracaibo del estado Zulia, las operaciones y la logística de entrada figuran como las actividades primarias más relevantes, mientras que el recurso humano y tecnológico asociado a las mismas comprenden las actividades de apoyo de mayor importancia.

En cuanto al dise ño de estrategias competitivas, comparando los resultados obtenidos en 1999 y 2003 se observa una tendencia hacia la selección de la diversificación como elemento fundamental a la hora de diseñar tales estrategias. Una porción importante de los entrevistados consideran una combinación de precio y nivel de diversificación. Por lo que, los resultados del estudio sugieren implantar para el sector una estrategia de diferenciación, con minimización del impacto en costos. Dada la cercanía de niveles de precios dentro de la cual compiten, la depresión del mercado local, aunado a la hipersensibilidad del consumidor hacia el precio, los pequeños confeccionistas se ven obligados a mantener un nivel de precios bajo, en este contexto resulta fundamental para la subsistencia, mantener una relación óptima precio-calidad, buscando atributos diferenciadores poco costosos pero de gran impacto en la decisión de compra del consumidor.

\section{Referencias Bibliográficas}

1. Ayala, Luis y Arias, Ramiro (2003), El análisis de la cadena de valor. Publicado en 
la pagina web: http://216.109.117. 135/search/cache? $p=$ cadena+de+ valor\&ei=utf8\&url=iVQI zb4lxgJ : www.3w3search.com/Edu/Merc/Es/ Fecha de la consulta: 12-07-2003 pp 1-8.

2. Fernández, Jainelly. Diario Panorama, 31-08-2003. Textileras se recuperarán más de $4 \%$ con las normas de etiquetado. Maracaibo, Venezuela. Cuerpo 2 pág. 5.

3. Instituto Nacional de Estadísticas INE, antes OCEI (1997). Principales Indicadores de la Industria Manufacturera 1988- 1997. Venezuela.

4. La Londe, Bernard (2000), Tiempo de integración. Revista Gestión Ene/Feb 2000. Publicado en la página web: http://www.ambitos.com.ar/art/00-1304-b-0.htm. Fecha de la consulta 12/07/2003. pp 1-6.

5. Monitor Company (1997), Creando las ventajas competitivas del sector textilconfección. Resumen Ejecutivo. Venezuela.

6. Porter, Michael (1998), Ventaja Competitiva. Creación y sostenimiento de un desempeño superior. México. Compañía editorial continental S.A. de C.V.. pp 7-243.

7. Porter, Michael (2000), El camino que conduce a la diferenciación. Revista Gestión Ene/Feb 2000. Publicado en la página web: http://www.ambitos.com.ar/art/ 00-1317b.htm. Fecha de la consulta 12/07/2003. pp 1-5.

8. Romero, Jenny; Sandrea, Maryana; Morales, Mariher; Boscán Mariby y Acosta, Ana (2000), La industria de la confección zuliana en la era de la competitividad. Revista Venezolana de Gerencia, año 5, No. 11. Venezuela. Vice Rectorado Académico de la Universidad del Zulia. Pp 189-208.

9. Sandrea, Maryana y Boscán, Mariby (2003), Informe final Proyecto: Desempeño económico de las PYMES manufactureras de la región zuliana. Adscrito al Programa: Factores de competitividad de la las PYMES manufactureras de la región zuliana.

10. Shank, John K. y Govindarajan, Vijay (1997), Gerencia Estratégica de Costos. La Nueva Herramienta para Desarrollar una Ventaja Competitiva. Colombia, editorial Norma S.A. pp 1-118. 